\title{
Safety of the paramedian supracerebellar-transtentorial approach for selective amygdalohippocampectomy
}

\author{
${ }^{*}$ Carlo Serra, MD, ${ }^{1}$ Kevin Akeret, MD, ${ }^{1}$ Victor E. Staartjes, BMed, ${ }^{1}$ Georgia Ramantani, MD, PhD, ${ }^{2}$ \\ Thomas Grunwald, MD, PhD, ${ }^{3}$ Hennric Jokeit, PhD, ${ }^{3}$ Julia Bauer, MSc, ${ }^{3}$ and \\ Niklaus Krayenbühl, MD ${ }^{1,4}$
}

\begin{abstract}
1Department of Neurosurgery, Clinical Neuroscience Center, University Hospital Zurich, University of Zurich; 'Division of Pediatric Neurology, University Children's Hospital, Zurich; ${ }^{3}$ Department of Neuropsychology, Swiss Epilepsy Clinic, Klinik Lengg AG, Zurich; and ${ }^{4}$ Division of Pediatric Neurosurgery, Children's University Hospital Zurich, Switzerland
\end{abstract}

\begin{abstract}
OBJECTIVE The goal of this study was to assess the reproducibility and safety of the recently introduced paramedian supracerebellar-transtentorial (PST) approach for selective amygdalohippocampectomy (SA).

METHODS The authors performed a retrospective analysis of prospectively collected data originating from their surgical register of patients undergoing SA via a PST approach for lesional medial temporal lobe epilepsy. All patients received thorough pre- and postoperative clinical (neurological, neuropsychological, psychiatric) and instrumental (ictal and long-term EEG, invasive EEG if needed, MRI) workup. Surgery-induced complications were assessed at discharge and at every follow-up thereafter and were classified according to Clavien-Dindo grade (CDG). Epilepsy outcome was defined according to Engel classification. Data were reported according to common descriptive statistical methods.
\end{abstract}

RESULTS Between May 2015 and May 2018, 17 patients underwent SA via a PST approach at the authors' institution (hippocampal sclerosis in 13 cases, WHO grade II glioma in 2 cases, and reactive gliosis in 2 cases). The median postoperative follow-up was 7 months (mean 9 months, range 3-19 months). There was no surgery-related mortality and no complication (CDG $\geq 2$ ) in the whole series. Transitory CDG 1 surgical complications occurred in 4 patients and had resolved in all of them by the first postoperative follow-up. One patient showed a deterioration of neuropsychological performance with new slight mnestic deficits. No patient experienced a clinically relevant postoperative visual field defect. No morbidity due to semisitting position was recorded. At last follow-up 13/17 (76.4\%) patients were in Engel class I $(9 / 17[52.9 \%]$ were in class IA).

CONCLUSIONS The PST approach is a reproducible and safe surgical route for SA. The achievable complication rate is in line with the best results in the literature. Visual function outcome particularly benefits from this highly selective, neocortex-sparing approach. A larger patient sample and longer follow-up will show in the future if the seizure control rate and neuropsychological outcome also compare better than those achieved with current common surgical techniques. https://thejns.org/doi/abs/10.3171/2020.1.FOCUS19909

KEYWORDS paramedian supracerebellar-transtentorial approach; selective amygdalohippocampectomy; epilepsy; safety; technique

$\mathrm{T}$ HE selective amygdalohippocampectomy (SA) is a surgical procedure indicated in cases of drug-resistant medial temporal lobe epilepsy in which the epileptogenic focus does not involve the temporal neocortex. First described by Niemeyer in $1958,{ }^{21}$ the procedure became popular after the seminal work of Wieser and Yaşargil, ${ }^{40}$ who described a proximal transsylvian-transamygdala approach allowing SA without trauma to the adjacent neocortical areas. ${ }^{23}$ Since then several variations have been described. A short but not exhaustive catalog would include several nuances of transcortical, transsulcal, and transcisternal approaches. ${ }^{12,17,22-24,28,29,34,37,41,42}$

In experienced hands, SA allows excellent surgical results with minimal morbidity. The reported seizure-free rate after $\mathrm{SA}$ ranges between $50 \%$ and $80 \%, 11,13,14,18,38,39$ whereas new neurological deficits range between $1.5 \%$ and

ABBREVIATIONS CDG = Clavien-Dindo grade; ILAE = International League Against Epilepsy; $\mathrm{PHG}=$ parahippocampal gyrus; $\mathrm{PST}=$ paramedian supracerebellar transtentorial; $S A$ = selective amygdalohippocampectomy; $T S=$ transverse sinus.

SUBMITTED November 29, 2019. ACCEPTED January 24, 2020.

INCLUDE WHEN CITING DOI: 10.3171/2020.1.FOCUS19909.

${ }^{*}$ C.S. and K.A. contributed equally to this work. 



FIG. 1. Stratigraphic depiction via brain specimen dissection of relevant anatomical structures involved in the PST approach. A: Supracerebellar view of the mediobasal temporal lobe after removal of the tentorium and vascular structures. B: Removal of the superior parietal lobule, precuneus, cuneus, and lingual on both sides. Decortication of the cingulate gyrus and PHG on the left side to show the course of their longitudinally running fibers. On the right side both gyri have been removed to expose the retrocommissural portion of the hippocampal formation. C: On the left side the dissection is brought forward as on the right side in panel B, whereas on the right side the splenial fibers have been removed to show the detailed course of the fimbria.

D: Supracerebellar close-up view of the mesencephalon and diencephalon. The whole hippocampus is now visible. On the right side the dentate gyrus has been removed. $\mathrm{cc}-\mathrm{s}=$ splenium of corpus callosum; $\mathrm{cg}=$ cingulate gyrus; $\mathrm{ch}-\mathrm{f}=$ choroidal fissure; $\mathrm{cos}$ = collateral sulcus; $\mathrm{dg}$ = dentate gyrus; $\mathrm{fg}$ = fusiform gyrus; $\mathrm{fi}=$ fimbria; hi- $\mathrm{h}=$ hippocampal head; ist = isthmus; Iv-a = atrium of the lateral ventricle; $p b=$ pineal body; $p g$ = parahippocampal gyrus; $p l x=$ plexus choroideus; th- $p=$ pulvinar thalami.

$8 \% .{ }^{8} \mathrm{New}$ visual field defects, which in the literature are often considered separately, range between $0 \%$ and $87 \% .43$ Performing an SA seems to be an important factor (together-among others-with the patient's age and disease laterality) for preserving neuropsychological function. ${ }^{9}$ Still, despite the minimally invasive nature of transsylvian $\mathrm{SA}$, in some centers trauma to neocortical structures remains an issue, particularly to visual pathways ${ }^{4,26-28}$ and to the so-called temporal stem. The recent introduction of the paramedian supracerebellar-transtentorial (PST) approach in 2012 by Professor Türe offered thus a brilliant solution to obviate the abovementioned issues. ${ }^{32}$ The PST approach offers a safe and direct route, medial to the collateral sulcus (see Fig. 1), well away from the neocortex, to remove selectively only meso- and allocortical structures. Preliminary experience with SA performed using the PST approach had been already presented by Türe et al. in their work from 2012. ${ }^{32}$ Issues regarding safety and reproducibility of the procedure have been raised by several authors (see Kaye in the comment to Kadri et al., ${ }^{15}$ or $\mathrm{Erdem}^{7}$ ). The PST approach for SA was introduced by the senior author (N.K.) at our institution in 2015 and has recently become our standard. The purpose of this study is thus to present our surgical results using the PST approach for SA, in order to show that this recently introduced procedure is safe, effective, and reproducible.

\section{Methods}

\section{Patient Population}

A consecutive series of patients undergoing the PST approach for SA at the Department of Neurosurgery of our institution (May 2015 onward) was evaluated. All patients undergoing SA for medial temporal lobe epilepsy 
are treated according to the same protocol, which includes long-term scalp video-EEG including ictal recording (including invasive diagnostic EEG if needed); preoperative and 3-month postoperative MRI with dedicated postprocessing protocol as previously detailed; ${ }^{1}$ and complete neurological (including manual perimetry), neuropsychological, and psychiatric pre- and postoperative evaluation performed by board-certified specialists. Clinical and EEG follow-ups are scheduled at 3, 6, and 12 months after surgery and then yearly thereafter in case of seizure control, or in appropriate intervals in case of recurrence. If needed, functional imaging and Wada test is also performed to ascertain hemispheric dominance. ${ }^{35,36}$ All patients undergo preoperative transthoracic echocardiography to detect any right-left shunt, which in our protocol is a contraindication for the sitting position. In patients with a right-left shunt the operation is performed with the patient in the lateral position. Clinical, surgical, and radiological data are prospectively collected and inserted in a surgical register as previously reported. ${ }^{25}$ Surgical complications were classified according to Clavien-Dindo grade (CDG) as follows: grade I, any deviation from the normal postoperative course not requiring surgical, endoscopic, or radiological intervention, but only basic medications; grade II, complications requiring drug treatments other than those allowed for grade I; grade III, complications requiring surgical, endoscopic, or radiological intervention; grade IV, life-threatening complications that require intensive care; and grade V, death of patient. ${ }^{5}$ Epilepsy outcome is defined according to Engel classification. ${ }^{6}$ Due to the small sample size, only the descriptive statistic is reported. This manuscript was compiled according to the Strengthening the Reporting of Observational Studies in Epidemiology (STROBE) statement. The registry was approved by our institutional committee, and data were treated according to the Declaration of Helsinki.

\section{Surgical Procedure}

Our surgical protocol resembles that originally described by Türe et al. in 2012. ${ }^{32}$ Briefly, the patient is placed in a semisitting position, with the head in a neutral position perpendicular to the floor and the hips and knees bent so that the lower legs of the patients are at approximately the same height as their head. After insertion of the probe for the transesophageal echocardiography, the surgical field is disinfected and draped. A paramedian linear incision between inion and asterion is performed, approximately one-third above and two-thirds below the linea nuchalis superior, far enough down to allow the height of the foramen magnum to be reached. The surgical microscope (OPMI Pentero; Zeiss) must be equipped with a short tube to reduce the distance between ocular tubes and the lens, making surgery with the patient in a sitting position more comfortable for the surgeon. Two burr holes are placed above the transverse sinus (TS), one approximately $6 \mathrm{~cm}$ from the other, and one approximately $2 \mathrm{~cm}$ above the TS in the midline. A craniotomy is performed down to the foramen magnum. Under the surgical microscope a $1-\mathrm{cm}$ dural incision is made at the caudal end of the exposure in order to release CSF from the cisterna magna. A semicircular concave incision is then made just under the TS, extending from one end of the exposure to the other. Bridging veins, if any, are mobilized and the tentorial incisura is reached in order to open the quadrigeminal plate cistern, allowing for further relaxing and passive opening of the transverse fissure. The tentorium is coagulated and a V-shaped incision going from the petrous ridge backward to the most posterior end of the exposure, and from there anteriorly to the free margin of the tentorium is performed. A suture is placed at the tentorial flap, which is then pulled downward, thereby opening the way to the supratentorial space. The collateral sulcus is identified laterally, and the ambient cistern medially, both delimiting the parahippocampal gyrus (PHG). A corticectomy is performed in the PHG at the level of the inferior colliculus, which marks the posterior limit of the resection, and subpial dissection is then carried forward until the temporal horn of the lateral ventricle is reached. Ultrasonic or suction-guided subpial dissection of the PHG is pushed forward to the uncus, with concomitant removal of the hippocampus up to the choroidal fissure. The choroidal fissure is opened and the hippocampal arteries and veins are identified, coagulated, and cut. Along the collateral eminence the hippocampus is detached from its lateral connection. Last, after removal of the hippocampus, the amygdala is removed subpially. Endoscopic inspection can now be performed to assure the completeness of the resection.

\section{Results}

Between May 2015 and May 2018, 17 patients underwent SA via a PST approach at our institution. Patient data are reported in Table 1. No patient had undergone previous epilepsy surgery. Ten patients had right-sided SA whereas 7 patients underwent an operation on the left side. The operation was performed in the sitting position in 11 cases, whereas the remainder were performed in the lateral position due to the right-left shunt. The median surgical duration (from Mayfield positioning to skin closure) was 245 minutes (mean 254 minutes, range 170-390 minutes). Histological analysis revealed a hippocampal sclerosis in 13 cases (International League Against Epilepsy [ILAE] class 1 in 5 cases, not further definable in 8); a WHO grade II glioma in 2 cases; and nonspecific reactive gliosis in 2 cases. The median postoperative follow-up was 7 months (mean 9 months, range 3-19 months).

\section{Safety: Incidence of Complications}

In the whole series there was no surgically induced mortality nor major surgical morbidity (defined as CDG $\geq 2$ ). At discharge, 4 patients $(23.5 \%)$ had a CDG 1 surgical complication: 3 patients had a partial trochlear nerve palsy, and 1 patient a partial oculomotor palsy. All of these deficits resolved in the immediate postoperative course so that at the first postoperative follow-up (3 months after operation) all patients were neurologically intact. At last follow-up, 1 patient showed a deterioration of neuropsychological performance with new slight memory deficits. Of particular interest, no patient experienced a clinically relevant postoperative visual field defect. Last, no morbidity due to the semisitting position was recorded. 
TABLE 1. Relevant anagraphic characteristics and complications in 17 patients with mesial temporal lobe epilepsy who underwent SA

\begin{tabular}{|c|c|c|c|c|c|c|c|c|c|c|c|c|c|}
\hline \multirow[b]{2}{*}{$\begin{array}{c}\text { Case } \\
\text { No. }\end{array}$} & \multicolumn{5}{|c|}{ Preop Patient Characteristics } & \multirow[b]{2}{*}{$\begin{array}{c}\text { Pathology \& } \\
\text { Histological Findings }\end{array}$} & \multirow[b]{2}{*}{ Side } & \multicolumn{2}{|c|}{ Complications } & \multicolumn{3}{|c|}{ Complication at Last FU } & \multirow{2}{*}{$\begin{array}{l}\text { Outcome } \\
\text { Engel } \\
\text { Class at } \\
\text { Last FU }\end{array}$} \\
\hline & Sex & $\begin{array}{l}\text { Age } \\
\text { (yrs) }\end{array}$ & $\begin{array}{l}\text { Neuro } \\
\text { Deficit }\end{array}$ & $\begin{array}{l}\text { Neuropsych } \\
\text { Deficit }\end{array}$ & $\begin{array}{c}\text { Psychiatric } \\
\text { Deficit }\end{array}$ & & & $\begin{array}{c}\text { At } \\
\text { Discharge }\end{array}$ & $\begin{array}{l}\text { At } \\
\text { Last } \\
\text { FU }\end{array}$ & $\begin{array}{c}\text { New } \\
\text { Neuro } \\
\text { Deficit }\end{array}$ & $\begin{array}{c}\text { New } \\
\text { Neuropsych } \\
\text { Deficit }\end{array}$ & $\begin{array}{c}\text { New } \\
\text { Psychiatric } \\
\text { Deficit }\end{array}$ & \\
\hline 1 & $\mathrm{~F}$ & 19 & No & Yes & Yes & Hippocampal sclerosis & Rt & No & No & No & No & No & IVA \\
\hline 2 & M & 17 & No & Yes & No & $\begin{array}{l}\text { Hippocampal sclero- } \\
\text { sis, ILAE } 1\end{array}$ & $\mathrm{Lt}$ & No & No & No & No & No & $\mathrm{IA}$ \\
\hline 3 & $\mathrm{~F}$ & 57 & No & Yes & No & Reactive gliosis & Rt & CDG I & No & No & No & No & IB \\
\hline 4 & $\mathrm{~F}$ & 67 & No & Yes & No & Reactive gliosis & $\mathrm{Lt}$ & No & No & No & No & No & IB \\
\hline 5 & $\mathrm{~F}$ & 36 & No & Yes & No & Diffuse glioma & Lt & No & No & No & No & No & $\mathrm{IA}$ \\
\hline 6 & $\mathrm{~F}$ & 48 & No & Yes & Yes & Hippocampal sclerosis & Rt & No & CDG I & No & Yes & No & IB \\
\hline 7 & M & 34 & No & Yes & No & Hippocampal sclerosis & $\mathrm{Lt}$ & No & No & No & No & No & $\mathrm{IA}$ \\
\hline 8 & $F$ & 53 & No & Yes & No & $\begin{array}{l}\text { Hippocampal sclero- } \\
\text { sis, ILAE } 1\end{array}$ & Rt & No & No & No & No & No & IB \\
\hline 9 & $\mathrm{~F}$ & 31 & Yes & Yes & Yes & Hippocampal sclerosis & Rt & CDG I & No & No & No & No & IA \\
\hline 10 & M & 33 & No & Yes & No & $\begin{array}{l}\text { Hippocampal sclero- } \\
\text { sis, ILAE } 1\end{array}$ & $\mathrm{Lt}$ & No & No & No & No & No & $\mathrm{IA}$ \\
\hline 11 & M & 33 & No & Yes & No & $\begin{array}{l}\text { Hippocampal sclero- } \\
\text { sis, ILAE } 1\end{array}$ & Rt & No & No & No & No & No & $\| \mathrm{A}$ \\
\hline 12 & M & 10 & No & No & No & Diffuse glioma & $\mathrm{Rt}$ & CDG I & No & No & No & No & IIB \\
\hline 13 & $\mathrm{~F}$ & 54 & Yes & Yes & Yes & $\begin{array}{l}\text { Hippocampal sclero- } \\
\text { sis, ILAE } 1\end{array}$ & Rt & No & No & No & No & No & IA \\
\hline 14 & $\mathrm{~F}$ & 28 & No & Yes & No & Hippocampal sclerosis & Rt & CDG I & No & No & No & No & IIB \\
\hline 15 & $M$ & 29 & No & No & No & Hippocampal sclerosis & Rt & No & No & No & No & No & $\mathrm{IA}$ \\
\hline 16 & M & 3 & No & No & No & Hippocampal sclerosis & $\mathrm{Lt}$ & No & No & No & No & No & IA \\
\hline 17 & $\mathrm{~F}$ & 26 & No & Yes & Yes & Hippocampal sclerosis & $\mathrm{Lt}$ & No & No & No & No & No & $\mathrm{IA}$ \\
\hline
\end{tabular}

$\mathrm{FU}=$ follow-up; neuro = neurological; neuropsych = neuropsychological.

\section{Seizure Outcome}

Table 1 shows the detailed outcomes of patients with respect to seizure control. At last follow-up 13/17 (76.4\%) patients were seizure free and thus allocated in Engel class I; $9 / 17(52.9 \%)$ patients were in class IA. Only 1 patient ended up in class IV, and is currently under evaluation for an extended resection. Two patients were able to stop taking antiepileptic medications.

\section{Discussion}

The current study aimed at assessing whether the recently introduced PST approach is a reproducible and safe approach for SA. The data resulting from our consecutive cohort of 17 patients allow an affirmative answer to the study question. Mortality and permanent morbidity were $0 \%$, with only 4 cases of transitory, short-lasting, CDG 1 complications. This result is even more relevant if one considers that the data originate from a prospective register, a fact that notoriously determines a higher incidence of reported complications. Moreover, the present study is one of the very few prospectively reporting complications in a standardized, reproducible manner, according to a widely adopted grading system ${ }^{5}$ that has been validated also for neurosurgical procedure ${ }^{2,43}$ including epilepsy surgery. ${ }^{28}$ As such, our results are particularly encouraging.

\section{Complication Rate and Safety of the PST Approach}

Reported complication rates in epilepsy surgery vary considerably in the literature, depending on the adopted technique, patients' characteristics, local expertise, and assessment criteria. According to recent data, permanent morbidity for temporal lobe epilepsy surgery has significantly dropped over the decades, from $42 \%$ in the very first series, down to $4 \%$ in the latest reports. ${ }^{28,30}$ Similar data seem to apply to SA. Permanent morbidity, excluding visual field defect, can be as low as $2.5 \%{ }^{27}$ if the procedure is performed via a transsylvian approach, although similar data are available also for transcortical and subtemporal approaches. ${ }^{8,28}$ Even though our data seem to compare favorably with the literature, the limited number of patients in our series does not allow us to conclude that the PST approach is safer than other surgical approaches. It allows us, however, to attest to the safety and feasibility of the approach.

The most striking advantage of the PST approach is probably the lower incidence of visual field defects, ${ }^{32}$ a finding that is confirmed by our study. Studies specifically addressing visual outcome show that the occurrence of a new postoperative visual field defect remains a considerable issue in epilepsy surgery for temporal lobe epilepsy. New visual field defects still occur in a substantial portion of patients; not only, as can be expected, in 
those undergoing transcortical approaches, but also in those undergoing transsylvian SA. In the series of Delev et al., ${ }^{4}$ some form of new visual field defect occurred in $87 \%$ of patients undergoing transsylvian SA and in 54\% of those undergoing SA via a so-called temporobasal approach. Schmeiser and colleagues found new visual field defects in $48 \%$ of their cohort. ${ }^{26,28}$ Indeed, as shown in anatomical studies based on white matter fiber dissection, ${ }^{15}$ disruption of the optic radiation can also occur with a transsylvian procedure if the corticectomy is placed more posterior than it should be, thus violating the limits of the amygdala. These anatomical data clearly explain the high incidence of visual field defects reported in the literature also after transsylvian SA. The PST approach on the contrary, requires resection of the PHG only. As such, the temporal neocortex together with the optic radiation is left untouched.

The lack of manipulation of the temporal neocortex as well as of the so-called temporal stem may represent an advantage also with regard to neuropsychological outcome. For the purpose of cognitive outcome, selective procedures are better than extensive temporal lobe resection. ${ }^{9,20}$ The removal of nonpathological functional tissue, as is the case of two-thirds temporal lobe resection, is associated with worse neuropsychological outcome. ${ }^{10}$ In our series only 1 patient experienced a neuropsychological deterioration (slight memory deficits).

\section{Technical Nuances: Pros and Cons of the PST Approach}

Compared to more common approaches, the PST represents a new surgical concept, which undisputedly requires advanced anatomical knowledge, microsurgical skills, and anesthesiological management. However, we believe that the necessary effort is worth the challenge, because the PST approach offers several advantages. Compared to transcortical approaches, it does not require any manipulation of otherwise healthy tissue. The PST approach allows a highly selective removal of the involved anatomical structure only, which in our view represents an unsurpassable "pro" of this approach. Intraoperative orientation is also much easier, with the limits of resection readily and clearly identifiable from the beginning of the procedure onward. This means that the surgeon has direct control over the effectiveness of surgery (i.e., the length of hippocampectomy) and does not need to rely on surrogates for it such as quantitative measurements or neuronavigation. In this regard, in our experience the PST approach compares favorably also with the transsylvian SA. Last, the PST approach allows surgeons to remove easily and in every case the whole of the hippocampal formation, from the uncus back to the level of the inferior colliculus, without the need of brain retraction or an extensive corticectomy.

The PST approach presents two theoretical limitations, both linked to the requirement of the semisitting position: surgeon discomfort and risk of air embolism. The former does not represent in our opinion a limitation given the short duration of the surgery, as long as the patient is properly positioned (as described in the Methods section). Air embolism has also never been a clinically relevant issue in the present series. Recent literature $3,16,19,31$ confirms that surgery in the semisitting position can be safely per- formed, provided that adequate anesthesiological management is done. Should the preoperative transthoracic echocardiography show a patent foramen ovale with the presence of a right-left shunt under Valsalva maneuver, then the approach can be performed also in the lateral position, as was the case in 6 patients in our series. A lateral position does not impede the correct execution of the surgical procedure, provided that some technical details are adequately addressed. The patient is positioned on the side contralateral to the lesion. Depending on the patient's habitus, the chest is elevated approximately $20^{\circ}$, the contralateral arm is laid on an armrest positioned under the level of the chest, whereas the ipsilateral arm is positioned on an armrest and gently pulled caudally. Particular attention must be paid to the head positioning, which must necessarily be adapted to the head and neck conformation of the patient. As a general rule, the head should be flexed as much as possible, rotated approximately $15^{\circ}-20^{\circ}$ toward the ipsilateral side (to avoid conflict with the ipsilateral shoulder) and then bent approximately $15^{\circ}-20^{\circ}$ toward the ipsilateral side.

\section{Seizure Outcome}

Given the short median follow-up ( $<24$ months), this study was not designed to obtain a conclusion about the superiority of the PST over other approaches regarding seizure outcome. Compared to most other techniques, the PST approach permits the surgeon to comfortably reach also the most posterior part of the hippocampus. For the purpose of seizure control, this feature is advantageous particularly for those with more posteriorly located hippocampal sclerosis, and may lead to improved seizure control rates. Our preliminary data $(76.4 \%$ of patients in Engel class I, $52.9 \%$ in Engel class IA) would suggest at least equipoise between the PST and the other available surgical approaches. Longer follow-up and the inclusion of more patients will allow us to make a more definitive conclusion in the future.

\section{Conclusions}

The PST approach offers an excellent route for SA. Provided there is adequate microsurgical training, the approach is reproducible and proved also in our experience to be safe. The general complication rate is in line with the best results available in the literature, as shown by data coming from our prospective register, with standardized complications assessment according to CDG. Visual outcome is better than what has been reported in the literature for other surgical approaches. A larger patient sample and longer follow-up will show in the future if the seizure control rate also compares better than that achieved with the current common surgical techniques.

\section{References}

1. Akeret K, Serra C, Rafi O, Staartjes VE, Fierstra J, Bellut $\mathrm{D}$, et al: Anatomical features of primary brain tumors affect seizure risk and semiology. Neuroimage Clin 22:101688, 2019

2. Bucher B, Maldaner N, Regli L, Sarnthein J, Serra C: Standardized assessment of outcome and complications in chron- 
ic subdural hematoma: results from a large case series. Acta Neurochir (Wien) 161:1297-1304, 2019

3. Choque-Velasquez J, Colasanti R, Resendiz-Nieves JC, Gonzáles-Echevarría KE, Raj R, Jahromi BR, et al: Praying sitting position for pineal region surgery: an efficient variant of a classic position in neurosurgery. World Neurosurg 113:e604-e611, 2018

4. Delev D, Wabbels B, Schramm J, Nelles M, Elger CE, von Lehe M, et al: Vision after trans-sylvian or temporobasal selective amygdalohippocampectomy: a prospective randomised trial. Acta Neurochir (Wien) 158:1757-1765, 2016

5. Dindo D, Demartines N, Clavien PA: Classification of surgical complications: a new proposal with evaluation in a cohort of 6336 patients and results of a survey. Ann Surg 240:205213, 2004

6. Engel J Jr, Van Ness P, Rasmussen TB, Ojemann LM: Outcome with respect to epileptic seizures, in Engel $\mathrm{J} \mathrm{Jr}$ (ed): Surgical Treatment of the Epilepsies, ed 2. New York: Raven Press, 1993, pp 609-621

7. Erdem A: Letter: The paramedian supracerebellar-transtentorial selective amygdalohippocampectomy for mediobasal temporal epilepsy. Oper Neurosurg (Hagerstown) 15:E33, 2018

8. Georgiadis I, Kapsalaki EZ, Fountas KN: Temporal lobe resective surgery for medically intractable epilepsy: a review of complications and side effects. Epilepsy Res Treat 2013:752195, 2013

9. Helmstaedter C: Cognitive outcomes of different surgical approaches in temporal lobe epilepsy. Epileptic Disord 15:221-239, 2013

10. Helmstaedter C, Roeske S, Kaaden S, Elger CE, Schramm $\mathrm{J}$ : Hippocampal resection length and memory outcome in selective epilepsy surgery. J Neurol Neurosurg Psychiatry 82:1375-1381, 2011

11. Hemb M, Palmini A, Paglioli E, Paglioli EB, Costa da Costa J, Azambuja N, et al: An 18-year follow-up of seizure outcome after surgery for temporal lobe epilepsy and hippocampal sclerosis. J Neurol Neurosurg Psychiatry 84:800-805, 2013

12. Hori T, Tabuchi S, Kurosaki M, Kondo S, Takenobu A, Watanabe T: Subtemporal amygdalohippocampectomy for treating medically intractable temporal lobe epilepsy. Neurosurgery 33:50-57, 1993

13. Hu WH, Zhang C, Zhang K, Meng FG, Chen N, Zhang JG: Selective amygdalohippocampectomy versus anterior temporal lobectomy in the management of mesial temporal lobe epilepsy: a meta-analysis of comparative studies. J Neurosurg 119:1089-1097, 2013

14. Josephson CB, Dykeman J, Fiest KM, Liu X, Sadler RM, Jette N, et al: Systematic review and meta-analysis of standard vs selective temporal lobe epilepsy surgery. Neurology 80:1669-1676, 2013

15. Kadri PAS, de Oliveira JG, Krayenbühl N, Türe U, de Oliveira EPL, Al-Mefty O, et al: Surgical approaches to the temporal horn: an anatomic analysis of white matter tract interruption. Oper Neurosurg (Hagerstown) 13:258-270, 2017

16. Klein J, Juratli TA, Weise M, Schackert G: A systematic review of the semi-sitting position in neurosurgical patients with patent foramen ovale: how frequent is paradoxical embolism? World Neurosurg 115:196-200, 2018

17. Kovanda TJ, Tubbs RS, Cohen-Gadol AA: Transsylvian selective amygdalohippocampectomy for treatment of medial temporal lobe epilepsy: surgical technique and operative nuances to avoid complications. Surg Neurol Int 5:133, 2014

18. Kuang Y, Yang T, Gu J, Kong B, Cheng L: Comparison of therapeutic effects between selective amygdalohippocampectomy and anterior temporal lobectomy for the treatment of temporal lobe epilepsy: a meta-analysis. Br J Neurosurg 28:374-377, 2014
19. Luostarinen T, Lindroos AC, Niiya T, Silvasti-Lundell M, Schramko A, Hernesniemi J, et al: Prone versus sitting position in neurosurgery-differences in patients' hemodynamic management. World Neurosurg 97:261-266, 2017

20. Malikova H, Kramska L, Vojtech Z, Liscak R, Sroubek J, Lukavsky J, et al: Different surgical approaches for mesial temporal epilepsy: resection extent, seizure, and neuropsychological outcomes. Stereotact Funct Neurosurg 92:372-380, 2014

21. Niemeyer P: The transventricular amygdala-hippocampectomy in the temporal lobe epilepsy, in Baldwin M, Bailey P (eds): The Temporal Lobe Epilepsy. Springfield, IL: Charles C Thomas, 1958, pp 461-82

22. Olivier A: Transcortical selective amygdalohippocampectomy in temporal lobe epilepsy. Can J Neurol Sci 27 (Suppl 1):S68-S76, S92-S96, 2000

23. Park TS, Bourgeois BFD, Silbergeld DL, Dodson WE: Subtemporal transparahippocampal amygdalohippocampectomy for surgical treatment of mesial temporal lobe epilepsy. Technical note. J Neurosurg 85:1172-1176, 1996

24. Rougier A, Saint-Hilaire JM, Bouvier G, Loiseau P, Baulac $\mathrm{M}$, Bouthillier A, et al: [Research and surgical treatment of epilepsy.] Neurochirurgie 38 (Suppl 1):1-112, 1992 (French)

25. Sarnthein J, Stieglitz L, Clavien PA, Regli L: A patient registry to improve patient safety: recording general neurosurgery complications. PLoS One 11:e0163154, 2016

26. Schmeiser B, Daniel M, Kogias E, Böhringer D, Egger K, Yang S, et al: Visual field defects following different resective procedures for mesiotemporal lobe epilepsy. Epilepsy Behav 76:39-45, 2017

27. Schmeiser B, Wagner K, Schulze-Bonhage A, Elger CE, Steinhoff BJ, Wendling AS, et al: Transsylvian selective amygdalohippocampectomy for mesiotemporal epilepsy: experience with 162 procedures. Neurosurgery 80:454-464, 2017

28. Schmeiser B, Wagner K, Schulze-Bonhage A, Mader I, Wendling AS, Steinhoff BJ, et al: Surgical treatment of mesiotemporal lobe epilepsy: which approach is favorable? Neurosurgery 81:992-1004, 2017

29. Shimizu H, Suzuki I, Ishijima B: Zygomatic approach for resection of mesial temporal epileptic focus. Neurosurgery 25:798-801, 1989

30. Tebo CC, Evins AI, Christos PJ, Kwon J, Schwartz TH: Evolution of cranial epilepsy surgery complication rates: a 32-year systematic review and meta-analysis. J Neurosurg 120:1415-1427, 2014

31. Türe H, Harput MV, Bekiroğlu N, Keskin Ö, Köner Ö, Türe $\mathrm{U}$ : Effect of the degree of head elevation on the incidence and severity of venous air embolism in cranial neurosurgical procedures with patients in the semisitting position. J Neurosurg 128:1560-1569, 2018

32. Türe U, Harput MV, Kaya AH, Baimedi P, Firat Z, Türe H, et al: The paramedian supracerebellar-transtentorial approach to the entire length of the mediobasal temporal region: an anatomical and clinical study. Laboratory investigation. J Neurosurg 116:773-791, 2012

33. Vadera S, Kshettry VR, Klaas P, Bingaman W: Seizure-free and neuropsychological outcomes after temporal lobectomy with amygdalohippocampectomy in pediatric patients with hippocampal sclerosis. J Neurosurg Pediatr 10:103-107, 2012

34. Vajkoczy P, Krakow K, Stodieck S, Pohlmann-Eden B, Schmiedek P: Modified approach for the selective treatment of temporal lobe epilepsy: transsylvian-transcisternal mesial en bloc resection. J Neurosurg 88:855-862, 1998

35. Wada J: A new method for the determination of the side of cerebral speech dominance. A preliminary report of the intra-carotid injection of sodium amytal in man. Igaku To Seibutsugaku 14:221-222, 1949 
36. Wada JA: A fateful encounter: sixty years later-reflections on the Wada test. Epilepsia 49:726-727, 2008

37. Wheatley BM: Selective amygdalohippocampectomy: the trans-middle temporal gyrus approach. Neurosurg Focus 25(3):E4, 2008

38. Wiebe S, Blume WT, Girvin JP, Eliasziw M: A randomized, controlled trial of surgery for temporal-lobe epilepsy. N Engl J Med 345:311-318, 2001

39. Wieser HG, Ortega M, Friedman A, Yonekawa Y: Long-term seizure outcomes following amygdalohippocampectomy. J Neurosurg 98:751-763, 2003

40. Wieser HG, Yaşargil MG: Selective amygdalohippocampectomy as a surgical treatment of mesiobasal limbic epilepsy. Surg Neurol 17:445-457, 1982

41. Yaşargil MG, Krayenbühl N, Roth P, Hsu SPC, Yaşargil DCH: The selective amygdalohippocampectomy for intractable temporal limbic seizures. J Neurosurg 112:168-185, 2010

42. Yaşargil MG, Teddy PJ, Roth P: Selective amygdalo-hippocampectomy operative anatomy and surgical technique, in Symon L, Brihaye J, Guidetti B, et al (eds): Advances and Technical Standards in Neurosurgery. Vienna: Springer, 1985, Vol 12, pp 93-123

43. Zattra CM, Zhang DY, Broggi M, Velz J, Vasella F, Seggewiss D, et al: Repeated craniotomies for intracranial tumors: is the risk increased? Pooled analysis of two prospective, institutional registries of complications and outcomes. J Neurooncol 142:49-57, 2019

\section{Disclosures}

The authors report no conflict of interest concerning the materials or methods used in this study or the findings specified in this paper.

\section{Author Contributions}

Conception and design: Serra, Akeret. Acquisition of data: Serra, Akeret, Staartjes, Ramantani, Grunwald, Jokeit, Bauer. Analysis and interpretation of data: Serra, Akeret, Jokeit. Drafting the article: Serra, Akeret. Critically revising the article: Serra, Akeret, Ramantani, Grunwald, Krayenbühl. Reviewed submitted version of manuscript: Serra, Akeret. Approved the final version of the manuscript on behalf of all authors: Serra. Statistical analysis: Serra, Akeret. Administrative/technical/material support: Serra, Akeret. Study supervision: Krayenbühl.

\section{Correspondence}

Carlo Serra: University Hospital of Zürich, University of Zürich, Switzerland.carlo.serra@usz.ch. 